Available online at<smiles>C1C2CC1C2</smiles>

www.sciencedirect.com
Elsevier Masson France

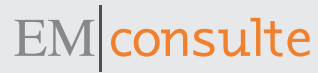

www.em-consulte.com

Original article

\title{
Anti-CCP antibodies are associated with early age at onset in patients with rheumatoid arthritis
}

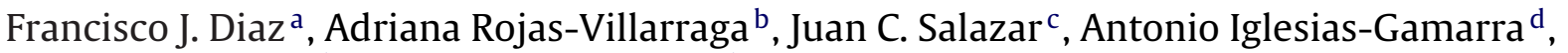 \\ Ruben D. Mantilla ${ }^{\mathrm{b}, \mathrm{e}}$, Juan-Manuel Anaya ${ }^{\mathrm{b}, *}$ \\ a Department of Biostatistics, the University of Kansas Medical Center, Kansas City, United States \\ b Center for Autoimmune Diseases Research (CREA), School of Medicine and Health Sciences, Universidad del Rosario, Carrera 24 \# 63C-69, Bogotá, Colombia \\ c Department of Statistics, Universidad Nacional de Colombia, Medellin, Colombia \\ d Rheumatology Unit, Universidad Nacional de Colombia, Bogotá, Colombia \\ e Rheumatology Unit, Riesgo de Fractura-CAYRE IPS, Bogotá, Colombia
}

\section{A R T I C L E I N F O}

Article history:

Accepted 8 July 2010

\section{Keywords:}

Rheumatoid arthritis

Age

HLA-DRB1

TNF-308 A allele

Rheumatoid factor

Anti-CCP antibodies

Smoking

\begin{abstract}
A B S T R A C T
Objectives: To determine factors influencing the age at onset of rheumatoid arthritis (RA)

Methods: A sample of 152 Colombian patients was investigated. Hazard ratios (HRs) that measured the effect size of risk factors on the age at RA onset were computed by using Cox regression models.

Results: Positive anti-CCP antibodies were associated with an increased risk of early RA onset $(\mathrm{HR}=1.60$; $95 \%$ confidence interval, $[1.06,2.4])$, whereas the presence of the protective ${ }^{70} \mathrm{DERAA}^{74}$ sequence was associated with a delayed onset ( $\mathrm{HR}=0.55$; $[0.33,0.92])$. After controlling for both anti-CCP antibodies and the ${ }^{70}$ DERAA $^{74}$ sequence, the following variables did not influence significantly the age at RA onset: gender, ever cigarette smoking, family RA history, the TNF-308 A polymorphism, HLA shared epitope, and the presence of rheumatoid factor.

Conclusion: Anti-CCP antibodies and the HLA-DRB1 ${ }^{70}$ DERAA $^{74}$ sequence influence the age at onset of RA. C 2010 Société française de rhumatologie. Published by Elsevier Masson SAS. All rights reserved.
\end{abstract}

\section{Introduction}

Age at onset of rheumatoid arthritis (RA) is influenced by genetic [1], hormonal [2], immunological and environmental factors [3]. Genetic risk factors are thought to be responsible for up to $50-60 \%$ of RA risk [4], and the HLA-DRB1 gene has been unequivocally associated with RA susceptibility. Anti-cyclic citrullinated peptide (anti-CPP) antibodies have received special attention, since they may be helpful for RA diagnosis; they are moderately sensitive but highly specific to RA, with a specificity that is higher than that of RF [5]. Anti-CCP antibodies can be detected at very early disease stages, and may be used as indicators of RA progression and prognosis [6]. The objective of the current study was to investigate the effects of demographic, antibody profile and immunogenetic characteristics on the age at onset of RA (AORA).

\footnotetext{
* Corresponding author. Tel.: +571 3474570 ext. 270; fax: +571 3499410

E-mail address: anayajm@gmail.com (J.-M. Anaya).
}

\section{Methods}

\subsection{Study population}

A sample of Colombian patients with RA was analyzed. The sample included 152 consecutive patients attending the Clinical Immunology and Rheumatology Unit at the "Clínica Universitaria Bolivariana-Corporación para Investigaciones Biológicas", Medellin, the Center for Autoimmune Diseases Research at the School of Medicine in the Rosario University, and the Rheumatology Unit at the "Universidad Nacional de Colombia" School of Medicine, Bogota, Colombia. All patients met the American College of Rheumatology criteria for RA classification [7] and had an AORA $\geq 17$ years. Only patients with a confirmed diagnosis of RA were included in the sample. Local institutional review boards and ethics committees approved the study, and all patients signed a written informed consent. A patients' description is in Table 1.

\subsection{Variables}

Data were recorded as previously reported $[8,9]$ and collected with a specifically created standard data collection form, which included demographic, clinical and laboratory variables. The AORA 
Table 1

Demographic and clinical characteristics of 152 Colombian patients with RA.

\begin{tabular}{|c|c|}
\hline Characteristics & Mean \pm SD \\
\hline Age at RA onset (years) & $42 \pm 14^{\mathrm{a}}$ \\
\hline \multirow[t]{2}{*}{ Disease duration (years) } & $8.5 \pm 7.3$ \\
\hline & $\%(n)$ \\
\hline Female gender & $85(129 / 152)$ \\
\hline Ever smoking ${ }^{c}$ & $22(33 / 152)$ \\
\hline \multicolumn{2}{|l|}{ Autoantibodies } \\
\hline Rheumatoid Factor & $73(103 / 141)$ \\
\hline Positive anti-CCP titers & $78(119 / 152)$ \\
\hline \multicolumn{2}{|l|}{ Genetic factors } \\
\hline Family history of RA & $7(10 / 151)$ \\
\hline Alleles encoding ${ }^{70}$ DERAA $^{74}$ & $11(17 / 152)$ \\
\hline HLA-DRB1 SE ${ }^{\mathrm{d}}$ & $51(78 / 152)$ \\
\hline TNF-308 $A$ allele & $26(38 / 149)$ \\
\hline
\end{tabular}

RA: rheumatoid arthritis; CCP: cyclic citrullinated peptide; HLA: human leukocyte antigen; SE: shared epitope; TNF: tumor necrosis factor gene; SD: standard deviation.

a The median, $25^{\text {th }}$ and $75^{\text {th }}$ percentiles were 40,32 and 52 years, respectively.

b Disease duration at moment of serological sampling.

c Ever smoking was assessed by a self-reported, validated questionnaire and was defined as having smoked at least one pack year in lifetime [7].

d Carrying at least one copy of any SE allele.

was defined as the age at which patients began to suffer from pain, morning stiffness, or a clinically confirmed inflammation of hand and/or foot joints in a symmetrical fashion. A family history of RA was defined as the clinically confirmed presence of the disease in at least one first-degree relative [6].

The TNF-308 A/G single nucleotide polymorphism (SNP) was genotyped by polymerase chain reaction (PCR) [10]. HLA-DRB1 typing was done by reverse dot-blot hybridization (Inno-LiPA assay) [11]. HLA-DRB1 status was categorized according to the presence of the shared epitope (SE) or ${ }^{70}{ }^{D E R A} A^{74}$ motifs, as described elsewhere $[9,11]$.

Total rheumatoid factor (RF) was measured by turbidimetry. An Elisa kit was used to measure third generation anti-cyclic citrullinated peptide (anti-CCP3) antibodies according to the manufacturer's protocol (Quanta-Lite, Inova, San Diego, CA).

\subsection{Statistical analysis}

A Cox regression model of AORA was built by using the SAS software [12] and a backward selection procedure. The AORA was the survival time. A model including all potential independent variables was initially fitted. Then the less significant variable was eliminated and the model was fitted with the remaining variables. This elimination continued until all variables in the model were significant at the 0.05 level [13]. The following were considered potential independent variables: being a carrier of the TNF-308 $A$ allele, carrying HLA-DRB1 SE alleles, the HLA ${ }^{70}$ DERAA $^{74}$ amino-acid sequence, positive RF titers ( $>40 \mathrm{U} / \mathrm{mL}$ ), positive antiCCP titers ( $>60 \mathrm{U}$ ), family history of RA, ever smoking, and female gender. Cox model goodness-of-fit was examined through logcumulative-hazard plots and Kolmogorov-type supremum tests. The final model fitted well. The size of the effect of a significant independent variable on AORA was measured as the hazard ratio corresponding to the variable [13]. Ninety-nine percent CIs for regression coefficients and hazard ratios were computed. Results with $P$-values $<0.05$ were considered significant.

\section{Results}

The mean \pm SD AORA was $42 \pm 14$ years (Table 1 ). Table 2 shows the variables significantly associated with AORA according to the Cox model. Positive anti-CCP3 antibodies were significantly associated with a lowered AORA after controlling for the presence of the HLA ${ }^{70}$ DERAA $^{74}$ sequence $(P=0.02$, Table 2$)$. Having a ${ }^{70}$ DERAA $^{74}$ sequence was significantly associated with a raised AORA after controlling for positive anti-CCP3 antibodies $(P=0.02)$. In patients who had the ${ }^{70} \mathrm{DERAA}^{74}$ sequence and were anti-CCP3 negative, the mean \pm SD AORA was $45.5 \pm 19.5$ years. In patients who had a ${ }^{70}$ DERAA $^{74}$ sequence but were anti-CCP3 positive, it was $51.4 \pm 13.3$ years. In patients who did not have a ${ }^{70}$ DERAA $^{74}$ sequence and were anti-CCP3 negative, it was $45.7 \pm 15.6$ years. In patients who did not have the ${ }^{70} \mathrm{DERAA}^{74}$ sequence and were anti-CCP3 positive, it was $39.5 \pm 12.1$ years.

The hazard ratio that compared the risk of RA onset in patients with positive versus patients of equal age with negative anti-CCP3 antibodies, adjusted for positive ${ }^{70} \mathrm{DERAA}^{74}$ sequence, was 1.60 (Table 2). Thus, at any particular age after 17 years, the hazard of RA onset for a patient with current positive anti-CCP3 antibodies was $60 \%$ higher than that for a patient with negative anti-CCP3 antibodies. Similarly, the hazard ratio that compared patients with the ${ }^{70}$ DERAA $^{74}$ sequence versus patients without it, adjusted by positive anti-CCP3 antibodies, was 0.55 . Therefore, at any particular age after 17 , the hazard of RA onset for a patient who had the ${ }^{70}$ DERAA $^{74}$ sequence was $45 \%$ lower than that for a patient who did not have it.

After controlling for both positive anti-CCP3 antibodies and ${ }^{70}$ DERAA $^{74}$ sequence, no other investigated variable was significantly associated with AORA. A trend between HLA-DRB ${ }^{*} 0404$ and positive anti-CCP3 antibodies was observed (Table 3). No significant differences in SE allele frequencies were observed between anti-CCP3 positive and negative patients ( $50 \%$ vs $55 \%$, respectively). There was a statistically significant difference in the frequencies of positive RF between anti-CCP3 positive and negative patients $(81 \%$ vs $41 \%$, respectively; $\mathrm{OR}=6.14 ; 95 \% \mathrm{CI}$, [2.55-14.77]; $P<0.001)$.

The mean \pm SD disease duration at moment of serological sampling was $8.5 \pm 7.3$ years. An additional Cox regression showed that there were not significant interactions between disease duration and the significant variables reported in Table 2 , and the hazard

Table 2

Variables significantly associated with the age at onset of rheumatoid arthritis (AORA) in Colombian patients with RA according to a Cox model.

\begin{tabular}{|c|c|c|c|c|c|}
\hline Variables $^{\mathrm{a}, \mathrm{b}}$ & $\beta^{c}$ & $95 \% \mathrm{CI}^{\mathrm{d}}$ & $P$-value & Hazard ratio & $95 \% \mathrm{CI}^{\mathrm{d}}$ \\
\hline Positive anti-CCP antibodies & 0.47 & $(0.006,0.88)$ & 0.02 & $1.60^{\mathrm{e}}$ & $(1.06,2.4)$ \\
\hline${ }^{70}$ DERAA $^{74}$ sequence & -0.59 & $(-1.10,-0.08)$ & 0.02 & $0.55^{\mathrm{f}}$ & $(0.33,0.92)$ \\
\hline
\end{tabular}

RA: rheumatoid arthritis; CCP: cyclic citrullinated peptide; $95 \% \mathrm{CI}$ : 95\% confidence interval.

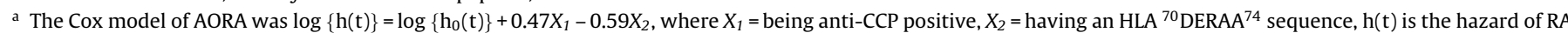

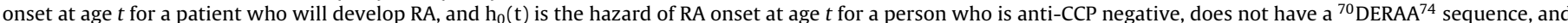
will develop RA (proportional hazards test for positive anti-CCP, $P=0.91$; for ${ }^{70}{ }^{D E R A A}{ }^{74}$ sequence, $P=0.90$ ).

b Independent variables included in the Cox model were dichotomous ( 1 = positive, $0=$ negative).

c The numbers in this column are Cox regression coefficients.

d $95 \%$ confidence interval for $\beta$ or the hazard ratio.

e The hazard ratio and its $95 \% \mathrm{CI}$ are adjusted for the presence of the ${ }^{70} \mathrm{DERAA}^{74}$ sequence.

f The hazard ratio and its $95 \% \mathrm{CI}$ are adjusted for positive anti-CCP antibodies. 
Table 3

Clinical and genetic characteristics of Colombian patients with RA stratified by the presence of anti-CCP antibodies status.

\begin{tabular}{|c|c|c|}
\hline Characteristics & Anti-CCP3 positive $(n=119)$ & Anti-CCP3 negative $(n=33)$ \\
\hline Female gender & $86(102 / 119)$ & $82(27 / 33)$ \\
\hline Ever smoking & $21(25 / 119)$ & $24(8 / 33)$ \\
\hline Family history of RA & $8(9 / 119)$ & $3(1 / 32)$ \\
\hline Reumatoid factor $(+)$ & $81(91 / 112)^{\mathrm{a}}$ & $41(12 / 29)$ \\
\hline Having the ${ }^{70}$ DERAA $^{74}$ sequence & $11(13 / 119)$ & $12(4 / 33)$ \\
\hline HLA-DRB $1 * 0404$ & $20(24 / 119)$ & $6(2 / 33)$ \\
\hline HLA-DRB $1 * 0401 / 0404$ & $0.8(1 / 119)$ & $0(0 / 33)$ \\
\hline HLA-DRB $1{ }^{70} \mathrm{QKRAA}^{74} \mathrm{SE}$ & $0.9(1 / 114)$ & $3(1 / 33)$ \\
\hline HLA-DRB $1{ }^{70}$ RRRAA $^{74}$ SE & $0(0 / 114)$ & $3(1 / 33)$ \\
\hline HLA-DRB $1{ }^{70} \mathrm{QRRAA}^{74} \mathrm{SE}$ & $47(54 / 114)$ & $48(16 / 33)$ \\
\hline HLA-DRB1 SE & $50(60 / 119)$ & $55(18 / 33)$ \\
\hline TNF-308 A allele & $26(30 / 117)$ & $25(8 / 32)$ \\
\hline
\end{tabular}

Data are in \% $(n)$. RA: rheumatoid arthritis; SE: shared epitope; CCP: cyclic citrullinated peptide.

a OR: $6.14(95 \% \mathrm{CI}=2.55-14.77), P<0.0001$. No other significant differences were observed, although a trend was observed in HLA-DRB1*0404 patients $(P=0.06)$.

b Carrying at least a copy of any SE allele.

ratios reported in the Table 2 were not substantially affected after including disease duration in the model.

\section{Discussion}

The main result of this study was that RA patients who are currently positive for anti-CCP antibodies developped RA at an earlier age than anti-CCP negative patients. This observation was established in a sample of Colombian patients, an admixed population from tropical latitudes. However, an association of anti-CCP antibodies with early RA onset was also found in 468 Swedish patients in whom anti-CCP antibodies were present in $67.9 \%$ of them [14]. In a sample of 411 Spanish patients with RA in which $54 \%$ were antiCCP positive, the trend for association observed between anti-CCP antibodies and age at onset disappeared when disease duration was taken into account [15].

Although important differences exist in the characteristics of patients reported in the literature as well as the cut-off value used to define a positive test, anti-CCP antibody testing is particularly useful in the diagnosis of RA, with high specificity [16]. Anti-CCP antibodies are usually present before the development of RA symptoms, suggesting that a break in tolerance to citrullination products occurs early in RA development [17]. Recent studies suggest that anti-CCP positive and negative RA might have different pathogenesis [18].

Having the ${ }^{70} \mathrm{DERAA}^{74}$ sequence protected against early RA onset in these Colombian patients. The ${ }^{70}$ DERAA $^{74}$ sequence alleles have been suggested to protect against RA onset and severity [19]. A model supported by some experimental and clinical studies, known as the RA-protection hypothesis, postulates that DQ molecules predispose to RA and that DR molecules $\left({ }^{70}\right.$ DERAA $\left.^{74}\right)$ could modulate disease severity by serving as a source of self-peptides presented in the context of DQ molecules [20]. The frequency of patients with RA carrying the ${ }^{70}$ DERAA $^{74}$ sequence ranges from 8 to $16 \%$ [20]; this variation is independent of the presence of HLA-DQ molecules or SE status. In the current study, this frequency was $11 \%$ (Table 1 ). Recently, the ${ }^{70}$ DERAA $^{74}$ sequence was reported to be a strong independent predictor of a better RA prognosis in a North-American early polyarthritis cohort [21].

A significant association between SE alleles and positive antiCCP3 antibodies was not detected in Colombian patients (Table 3 ), despite the fact that significant associations have been reported in the published literature. This lack of association may be caused by an insufficient sample size or by a modest effect of HLA on the synthesis of anti-CCP antibodies in our population since the influence of genotype on phenotype varies across populations [6].

Although individuals with an RA family history or HLA-DRB1 risk alleles are considered to be at high risk of developing RA [22,23], our study did not find an association between these variables and an early AORA. Our study did not find a significant effect of ever smoking on AORA after adjusting for significant variables, although an effect of current cigarette smoking on AORA has been previously reported [3].

In conclusion, anti-CCP antibodies and the HLA-DRB1 ${ }^{70} \mathrm{DERAA}^{74}$ sequence influence the age at onset of RA in Colombian patients. Prospective studies are needed to examine whether this finding may be useful to predict an early RA onset in subjects genetically prone to RA, and to design strategies for preventing early RA onset as well.

\section{Conflict of interest statement}

The authors report no conflicts of interest.

\section{Acknowledgments}

This work was supported by Colciencias (1101-04-14156, to AIG, and 186/2007 to FJD and JCS); the Universidad Nacional de Colombia(DIB-809275); an unrestricted grant from Lafrancol SAS, and the School of Medicine and Health Sciences, Universidad del Rosario, Bogotá, Colombia. We are indebted to Tom W.J. Huizinga for fruitful discussion and criticism, and to Oscar Ortega for his assistance.

\section{References}

[1] MacGregor A, Ollier W, Thomson W, et al. HLA-DRB1*0401/0404 genotype and rheumatoid arthritis: increased association in men, young age at onset, and disease severity. J Rheumatol 1995;22:1032-6.

[2] Goemaere S, Ackerman C, Goethals K, et al. Onset of symptoms of rheumatoid arthritis in relation to age, sex and menopausal transition. J Rheumatol 1990;17:1620-2.

[3] Hutchinson D, Lynch MP, Moots RJ, et al. The influence of current cigarette smoking on the age at onset of rheumatoid arthritis (RA) in individuals with sporadic and familial RA. Rheumatology (Oxford) 2001;40:1068-70.

[4] MacGregor AJ, Snieder H, Rigby AS, et al. Characterizing the quantitative genetic contribution to rheumatoid arthritis using data from twins. Arthritis Rheum 2000;43:30-7.

[5] Caro-Oleas JL, Fernández-Suárez A, Reneses Cesteros S, et al. Evaluation of third generation anti-CCP antibodies in the diagnosis of rheumatoid arthritis from undifferentiated polyarthritis after 4 years of follow-up. Clin Exp Rheumatol 2008;26:461-3.

[6] Rojas-Villarraga A, Diaz FJ, Calvo-Páramo E, et al. Familial disease, the HLADRB1 shared epitope and anti-CCP antibodies influence time at appearance of substantial joint damage in rheumatoid arthritis. J Autoimmun 2009;32: 64-9.

[7] Arnett FC, Edworthy SM, Bloch DA, et al. The American Rheumatism Association 1987 revised criteria for the classification of rheumatoid arthritis. Arthritis Rheum 1988;31:315-24.

[8] Anaya JM, Correa PA, Mantilla RD, et al. Rheumatoid arthritis in African Colombians from Quibdo. Semin Arthritis Rheum 2001;31:191-8.

[9] Rojas-Villarraga A, Ortega-Hernandez OD, Gomez LF, et al. Risk factors associated with different stages of atherosclerosis in Colombian patients with rheumatoid arthritis. Semin Arthritis Rheum 2008;38:71-82. 
[10] Correa PA, Gomez LM, Cadena J, et al. Autoimmunity and tuberculosis. Opposite association with TNF polymorphism. J Rheumatol 2005;32:219-24.

[11] Anaya JM, Correa PA, Mantilla RD, et al. Rheumatoid arthritis association in Colombian population is restricted to HLA-DRB1*04 QRRAA alleles. Genes Immun 2002;3:56-8.

[12] Allison PD. Survival analysis using the SAS system: a practical guide. Cary, NC: SAS Institute Inc; 1998.

[13] Woodward MW. Epidemiology. Study design and data analysis. Boca Raton: Chapman \& Hall/C; 1999.

[14] Kokkonen H, Johansson M, Innala L, et al. The PTPN22 1858C/T polymorphism is associated with anti-cyclic citrullinated peptide antibody-positive early rheumatoid arthritis in northern Sweden. Arthritis Res Ther 2007;9:R56.

[15] Varadé J, Loza-Santamaría E, Fernández-Arquero M, et al. Shared epitope and anti-cyclic citrullinated peptide antibodies: relationship with age at onset and duration of disease in rheumatoid arthritis. J Rheumatol 2009;36:1085-6.

[16] Avouac J, Gossec L, Dougados M. Diagnostic and predictive value of anti-cyclic citrullinated protein antibodies in rheumatoid arthritis: a systematic literature review. Ann Rheum Dis 2006;65:845-51.

[17] Nielen MM, van Schaardenburg D, Reesink HW, et al. Specific autoantibodies precede the symptoms of rheumatoid arthritis: a study of serial measurements in blood donors. Arthritis Rheum 2004;50:380-6.
[18] van Oosterhout M, Bajema I, Levarht EW, et al. Differences in synovial tissue infiltrates between anti-cyclic citrullinated peptide-positive rheumatoid arthritis and anti-cyclic citrullinated peptide-negative rheumatoid arthritis. Arthritis Rheum 2008;58:53-60.

[19] Van der Helm-van Mil AH, Huizinga TW, Schreuder GM, et al. An independent role of protective HLA class II alleles in rheumatoid arthritis severity and susceptibility. Arthritis Rheum 2005;52:2637-44.

[20] de Vries R, Huizinga TWH, Toes REM. HLA and RA revisited: citrullinated food for the SE hypothesis, the DR6 effect, and NIMA. Human Immunol 2006;67: 454-9.

[21] Carrier N, Cossette P, Daniel C, et al. The DERAA HLA-DR alleles in patients with early polyarthritis: protection against severe disease and lack of association with rheumatoid arthritis autoantibodies. Arthritis Rheum 2009;60: 698-707.

[22] Gregersen PK, Silver J, Winchester RJ. The shared epitope hypothesis. An approach to understanding the molecular genetics of susceptibility to rheumatoid arthritis. Arthritis Rheum 1987;30:1205-13.

[23] Lin JP, Cash JM, Doyle SZ, et al. Familial clustering of rheumatoid arthritis with other autoimmune diseases. Hum Genet 1998;103: 475-82. 